\title{
Investigation and Countermeasures of Medical Staff's Demand for Nursing Workers in Chifeng
}

\author{
Tianliang Cui* \\ Internal Medicine-Neurology, Chifeng Municipal Hospital \& Chifeng Clinical Medical School of Inner Mongolia Medical \\ University, Chifeng 024000, Inner Mongolia Autonomous Region, China \\ *Corresponding author: Tianliang Cui, cuitianliang1983@126.com

\begin{abstract}
In order to understand the current demand of the medical staffs for nursing workers in Chifeng and to explore the practical management mode of nursing workers, 150 doctors and nurses were randomly selected from general tertiary hospitals in Chifeng to participate in a questionnaire survey. The objective of this study was to investigate the demand of nursing workers in tertiary hospitals in Chifeng and the satisfaction of medical staffs toward the existing nursing work. The results of the investigation were analyzed to provide countermeasures and suggestions for the nursing work in Chifeng.
\end{abstract}

Keywords: Medical staff; Demand for nursing workers; Chifeng; Investigation; Countermeasures

Publication date: November 2021; Online publication: November 30, 2021

\section{Introduction}

According to the data in the National Bureau of Statistics, the number of elderly people aged 60 years or above in China was 249 million at the end of 2018, accounting for $17.9 \%$ of China's total population ${ }^{[1]}$. The study found that half of the elderly people in China have at least one chronic disease and nearly one fifth of the elderly people have at least two kinds of chronic diseases ${ }^{[2]}$. The death caused by chronic diseases accounts for $88 \%$ of the total number of deaths in China. Therefore, the aging population is not only a change in the population structure, but also a decrease in the overall health level of the elderly and an increase in the prevalence of chronic diseases, thus increasing the burden on families and society. As a result, the pressure of providing for the aged in China is increasing, especially with the increase of "onlychild" families, the demand of patients and their families for nursing would also increase. No matter for the country or for the family, there is great pressure and burden. Chifeng, as the most populated city in Inner Mongolia Autonomous Region, has a total population of 4.518 million ${ }^{[3-5]}$. It also faces great pressure in terms of pension and care. At present, it has become a social problem, and the social demand for nursing workers is increasing. However, looking at the overall situation in Chifeng, nursing workers have formed a group, most of them do not have a health certificate neither have they received any professional training from the hospital; hence, they are only able to do some simple nursing. The nursing care for patients is not standardized, and the charges are not unified. There are times when they bid up the prices at will, raise the charges ( 3 times the salary), and compete with the patients in the ward maliciously, especially during the holidays. As a result, many patients with mild illnesses are unable to hire a nurse, many patients with severe illness are unable to hire a good nurse, and patients with poor economic conditions are unable to afford a nurse. Therefore, it is of practical significance to understand the current situation of the needs for nursing workers in Chifeng and to explore the practical management mode of nursing workers, so as to improve the quality of nursing workers and meet the needs of the society. 


\section{Materials and methods}

\subsection{Research subjects}

A total of 150 doctors and nurses were randomly selected from the top three hospitals in Chifeng to participate in a questionnaire survey. The objective of this study was to investigate the demand for nursing workers in tertiary hospitals in Chifeng and the satisfaction of medical staffs toward the existing nursing work.

\subsection{Methods}

In this study, a field questionnaire survey was used, and trained nurses served as investigators. The investigators visited the wards and various departments in the hospitals for investigation. Questionnaires were distributed to investigate the satisfaction of medical staffs toward the current situation of nursing workers. The questionnaire consisted of three parts: the first part comprised of questions to gather the basic information of the medical staffs, such as gender, professional title, education background, work position, department, administrative position, etc.; the second part was about the current and past employment of nursing workers as well as the understanding of the necessity of employing nursing workers; the third part was about the satisfaction toward nursing workers, mainly to understand the service attitude, work ability, service quality, professional ethics, work cooperation, and work execution of the medical staffs.

\subsection{Quality control}

The respondents were the medical staffs from general tertiary hospitals in Chifeng, who had studied health statistics, social survey methods, and other courses during their years in university. The investigators participated in case analysis and group project research of health service management, so they understood the significance of statistical analysis. This survey can well control the quality of the investigation.

\section{Results}

\subsection{Basic information}

Through a 5-month questionnaire survey, 150 questionnaires were collected from general tertiary hospitals in Chifeng. Among them, 149 questionnaires were valid, with an effective rate of $99.33 \%$. The basic information of the respondents is shown in Table 1.

Table 1. Basic information of respondents

\begin{tabular}{llc}
\hline Item & Information & Number of people \\
\hline \multirow{2}{*}{ Gender } & Woman & 145 \\
\cline { 2 - 3 } Management role & Man & 4 \\
\hline \multirow{3}{*}{ Education } & Yes & 15 \\
\cline { 2 - 3 } & No & 134 \\
\hline \multirow{3}{*}{ Department } & Junior college & 9 \\
\cline { 2 - 3 } & Undergraduate & 132 \\
\cline { 2 - 3 } & Graduate student & 8 \\
\hline & Internal medicine & 121 \\
\cline { 2 - 3 } & Surgery & 22 \\
\cline { 2 - 3 } & Functional & 3 \\
\cline { 2 - 3 } & Other & 3 \\
\hline
\end{tabular}




\subsection{Statistics of the survey results}

According to the survey results, the medical staffs from various departments felt that the inpatients have a high demand for nursing workers, especially critical patients as well as those with difficulties and in need of assistance. At the same time, in order to better manage the department, there is also a demand for nursing workers. The details are shown in Figure 1 and Figure 2.

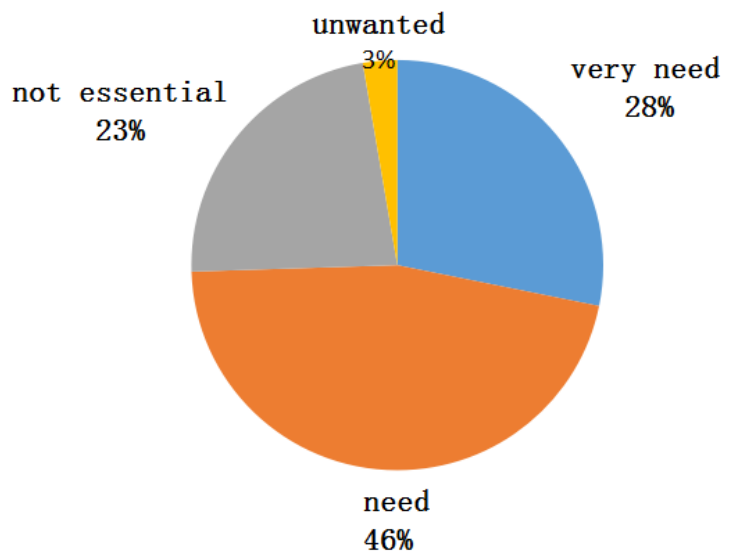

Figure 1. Demand of medical staffs for nursing workers

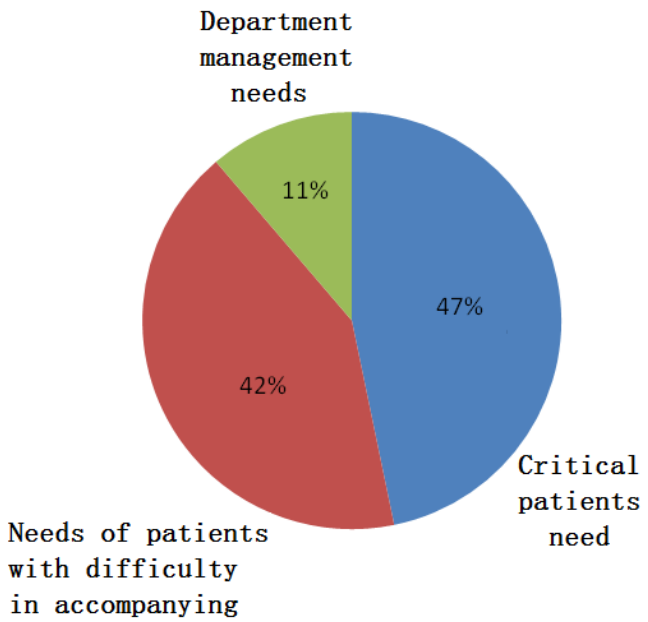

Figure 2. Reasons for the need of nursing workers based on the medical staffs

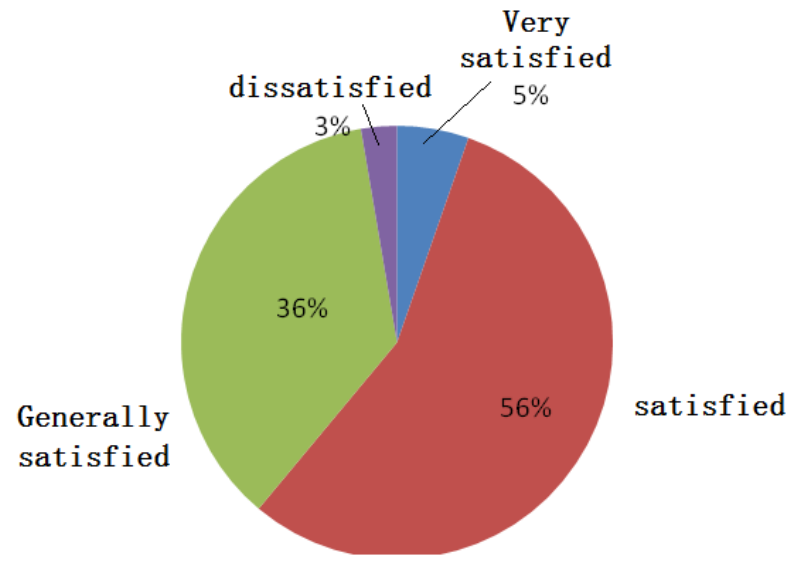

Figure 3. Satisfaction of medical staffs toward nursing workers 
As shown in Figure 3, the medical staffs were generally satisfied with the service attitude, work ability, work quality, professional ethics, work cooperation degree, and work execution of the nurses in the department; however, some dissatisfaction still exist among the medical staffs.

\subsection{Soliciting opinions}

From the third part of the questionnaire, comprising of the opinions and suggestions of the medical staffs on the work of nursing workers, it can be seen that most of the medical staffs felt that the existing nursing team is not well-standardized, and their working ability is uneven, with a lot of room for improvement. For example, some nursing workers have poor professional ability and their professional quality is low; they are not able to provide care for critical patients as observed by the medical staffs. At the same time, from the perspective of the medical staffs, the management of nursing workers is chaotic; there is no unified standard, standardized training, or supervision mechanism for the nursing workers. In addition, the labor cost of nursing workers is too high. Most people are unable to afford to hire nursing workers as the economic burden is too much to bear.

\section{Countermeasures and suggestions}

\subsection{Establishing an integrated model of "hospital-nurse-company"}

In the past, Chifeng was chaotic as there were large numbers of unattended private nursing workers, resulting in vicious competitions; this had seriously disrupted the market order. According to the survey, the patients' family members have higher recognition for the nursing workers from the hospital. It has been suggested that tertiary hospitals should try to cultivate their own nursing workers based on the needs of nursing workers and set up their own nursing teams. As there are adequate teachers in nursing training hospitals, after training and being qualified through assessment, the nursing staffs would be more professional, more standardized, and more in place compared to the former nursing workers. Upon reporting to duty, they would be managed by the department in a unified way, and they would receive special skills training from professional departments. In the hospital, the nursing workers work under the supervision of nurses to ensure the quality of patient care.

\subsection{Standardizing the supervision, assessment, and incentive mechanism of nursing workers}

In the past, there was a lack of supervision on nursing workers, and their work was more casual. The quality of work depended entirely on their professional ethics, and there was no assessment mechanism then. This was one of the reasons for the confusion and price hike of nursing workers. In order to promote the professional construction of nursing workers, nursing companies, hospitals, and patients should strengthen the supervision of nursing workers. Nurses with poor sense of responsibility and professional ability should be encouraged. Workers should provide timely feedback, especially for critically ill patients, and timely replace the nurses with strong sense of responsibility and excellent technical ability, so as to ensure that they can cooperate with the medical treatment and nursing of patients, thus providing better care. Other than that, impromptu assessments and satisfaction surveys can also be carried out. For excellent employees, incentives, bonuses, or honor should be given. Nursing workers should be rated based on a comprehensive evaluation of their abilities from all aspects. Different patients should then be allocated based on the grading of those nursing workers. Different levels of wages and treatment should be implemented to encourage them to learn continuously. At the same time, it is also necessary to develop career planning programs for nursing workers, so that excellent nursing workers can be promoted to managers, continuously mobilize their enthusiasm, and attract more young and highly educated college and secondary school nursing graduates to join the nursing team, so as to improve the professional level and professional quality of the 
nursing industry. In assisting their career planning, a flow mechanism from nursing workers to nurses can be established for outstanding nursing workers who graduated from nursing profession, so as to improve the professional construction of the whole nursing industry.

\section{Conclusion}

With aging population, Chifeng, as the largest populated city in Inner Mongolia, has a large gap of nursing workers, and the demand for nursing workers is becoming more and more prominent. Through investigating the demand of medical staffs for nursing workers in Chifeng, opinions and suggestions have been proposed to solve the problems among nursing workers. The two suggestions include the establishing of an integrated model of "hospital-nurse-company" and the standardization of the supervision, assessment, as well as incentive mechanism for nursing workers.

\section{Disclosure statement}

The author declares that there is no conflict of interest.

\section{References}

[1] Guo S, Han L, Li Y, et al., 2019, Study on the Change Characteristics and Prevention Strategies of Chronic Diseases in the Elderly. Report on the Development of Active Response to Population Aging in the New Era, 2019: 47-49.

[2] Wu J, 1997, Clinical Effect of Nurse Management. Journal of Practical Nursing, 13(11): 606-607.

[3] Wang Y, Ruan L, Shi D, et al., 2011, Practice and Thinking of Standardized Management of Nursing Workers in Large General Hospitals. Chinese Hospital, 15(5): 70-72.

[4] Liu L, Liu W, Wang J, 2011, Work Autonomy of Certified Nursing Assistants in Long-Term Care Facilities: Discrepant Perceptions Between Nursing Supervisors and Certified Nursing Assistants. J Am Med Dir Assoc, 12: 524-534.

[5] Xu Y, 2008, Load Bearing Turning of Nursing Workers. President of Chinese Hospital, 2008(15): 6266. 